\title{
Platelets (2018)
}

\section{Platelet count correlates with stage and predicts survival in melanoma}

Saleh Rachidi, MD, PhD ${ }^{1, *}$, Maneet Kaur, MPH ${ }^{2}$, Tim Lautenschlaeger, MD ${ }^{3}$, Zihai Li, MD, $\mathrm{PhD}^{4}$

${ }^{1}$ Resident Physician, Department of Dermatology, Johns Hopkins University, Baltimore, MD, USA

${ }^{2} \mathrm{PhD}$ student in Biostatistics and Epidemiology, Bloomberg School of Public Health, Johns Hopkins University, Baltimore, MD, USA

${ }^{3}$ Assistant Professor, Department of Radiation Oncology, Indiana University, Indianapolis, IN, USA

${ }^{4}$ Professor and Chair, Department of Microbiology and Immunology, Medical University of South Carolina, Charleston, SC, USA

${ }^{*}$ Corresponding author: 1550 Orleans Street, Suite 211, Baltimore, MD 21231, Email: salehrachidi@gmail.com

Word count: Abstract; 194. Text; 2,157.

This paper includes 3 tables, 2 figures, 5 supplementary tables, and 21 references.

This work was approved by the IRB at Indiana University.

The authors have no conflicts of interest to declare. Funding sources: None.

Running head: Platelets, stage and survival in melanoma

This is the author's manuscript of the article published in final edited form as:

Rachidi, S., Kaur, M., Lautenschlaeger, T., \& Li, Z. (2019). Platelet count correlates with stage and predicts survival in melanoma. Platelets, 1-5. https://doi.org/10.1080/09537104.2019.1572879 


\section{Abstract}

Cancer is a chronic inflammatory state which is often associated with increased platelet counts. Cancer cells induce thrombopoiesis and activate platelets, which in turn facilitate cancer invasion and metastasis. In this study, we investigate the correlation between platelet counts with each of stage and overall survival in melanoma. This is a retrospective cohort study of 642 melanoma patients diagnosed or treated at a tertiary medical center between 2000 and 2016. Multivariable analysis adjusted for age, sex, stage and treatment modality. Using multivariable analysis, patients with thrombocytosis around time of diagnosis were more likely to present with distant metastasis (Prevalence Ratio 3.5, 95\% $\mathrm{Cl}$ 2.35-5.22). In patients with metastatic disease and in all stages combined, thrombocytosis predicted decreased 5-year overall survival in univariate and multivariable analysis, and this was most pronounced during the first year from diagnosis. Finally, we show that mice with thrombocytopenia due to the lack of heat shock protein gp96 in their megakaryocytes are protected from melanoma dissemination to the lungs. These findings are concordant with preclinical studies showing a role for platelets in cancer metastasis and suppression of antitumor immunity, further supporting targeting platelets as an adjuvant to immunotherapy in melanoma.

Key words: Melanoma, platelets, stage, survival. 


\section{Introduction}

In addition to their role in hemostasis, platelets contain a myriad of bioactive molecules and contribute to inflammation, cancer invasion and metastasis. Tumor cells engage platelets in a process termed tumor cell-induced platelet aggregation (TCIPA), which aids the former in evading the immune system[1]. Cancer cell-derived thrombin is a mediator of this process and facilitates metastasis[2], and the ability of cancer cells to initiate TCIPA correlates with their metastatic potential[3]. Moreover, tumor-derived IL-6 induces thrombopoietin production by the liver, thus promoting thrombopoiesis[4], at least partially explaining the association between thrombocytosis and malignancy[5].

When cancer cells leave the primary tumor and enter blood stream, they activate surrounding platelets via molecules such as HMB1[6] and ADP[7], inducing microthrombus formation. This in turn shields cancer cells from the immune attack by natural killer cells[7]. Mice with defective platelet function are protected from melanoma dissemination to their lungs; however, this protection is mitigated by depletion of natural killer cells[8]. Platelets also propagate tumor cell metastasis independent of natural killer cells[9]. CD97 on cancer cells activates platelets to release lysophosphatidic acid (LPA), thus facilitating trans-endothelial migration[10]. We have also shown that platelets activate TGF $\beta$ and suppress anti-tumor T lymphocytes, thus aiding in melanoma growth[11]. Platelet-derived TGF $\beta$ also acts directly on cancer cells inducing epithelialmesenchymal transition, thus increasing their metastatic potential[12]. Moreover, platelets secrete CXCL5 and CXCL7 which recruit granulocytes to cancer cells, leading to the formation of early metastatic niches[13].

In recent work, we showed that aspirin use after diagnosis correlated with longer survival in advanced stages of melanoma. We also observed that aspirin intake prior to diagnosis correlated with earlier stages[14]. In addition, increased levels of circulating platelet-derived microparticles are associated with metastatic cutaneous melanoma[15]. Increased platelet counts have also 
been associated with worse outcomes in other cancer types[16, 17, 18]. In light of this evidence, we investigate here the association between platelet count and stage of melanoma, as well as the prognostic value of platelet count.

\section{Methods}

\section{Study design}

This study was approved by the institutional review board (IRB) at Indiana University (IU). We obtained demographic and cancer-related data from the Simon Comprehensive Cancer Center registry at IU. Data are collected by the registry from pathology reports, electronic medical records, radiation and chemotherapy oncology reports, and coding and billing data. Patients in this study were diagnosed between January $1^{\text {st }}, 2000$ and December $31^{\text {st }}, 2014$, and followed-up through September, 2016. Patients with platelet counts obtained within 3 months from date of diagnosis and prior to any systemic treatment, and with complete TNM staging were included in our analysis. The vast majority (99\%) of the patients were Caucasian. Our analysis was thus limited to Caucasian patients since the small number of other racial groups limits the generalizability of our findings to non-Caucasian patients, and prohibits adjusting for race in multivariable analysis. However, we performed sensitivity analyses to demonstrate that including other racial groups does not change our findings. Overall survival was calculated as the interval from the time of diagnosis to date of last contact, and survival times for patients alive at time of last contact were censored. For each patient, we obtained data on platelet counts from the IU Clinical Data Warehouse (CDW). Thrombocytosis was defined as platelet count $>350 \times 10^{3} / \mu \mathrm{L}$.

\section{Data analysis}

Descriptive statistics were used to summarize continuous and discrete variables. Continuous variables were compared using two-sided t-test and discrete variables were compared using the 
chi-square or two-sided Fisher's exact test, depending on the pertinent sample size. All statistical analyses were done using Stata version 14.1.

We used Kaplan-Meier plots to visualize univariate survival after melanoma diagnosis by platelet count, and survival curves were compared statistically using log-rank tests. We estimated the association between platelet counts and melanoma survival as hazard ratios and $95 \%$ confidence intervals from Cox proportional hazards regression with time from diagnosis as the time metric. We examined the proportional hazards assumption using Schoenfeld residuals, visually using log-log survival plots, and by modeling interaction terms between platelet counts and time. We found evidence for non-proportionality in analysis of all stages combined and stage 4 melanoma, specifically occurring early after diagnosis, so we reported hazard ratios for 1-year survival and 5-year survival, conditional on 1-year survival.

We used log-binomial regression to estimate prevalence ratios and $95 \%$ confidence intervals between platelet count and stage. Our outcome of interest for stage was stage IV versus stage 0-III. When log-binomial model failed to converge, we used Poisson regression with robust variance, which approximates the log-binomial estimates.

Platelet counts were available in $40.5 \%$ of participants. Due to potential selection bias using only participants with platelet counts, we performed a sensitivity analysis, repeating all analyses using multiple imputation to account for missingness (Supplementary material). We assumed the platelet counts were missing at random and that missingness could be explained by variables in our dataset. We examined predictors of platelet count and missingness to determine the imputation model and decided upon using the following variables: age at diagnosis, stage, diabetes, hypertension, alcohol use, vital status, and time to death or censoring. We used multiple imputation with Markov Chain Monte Carlo (MCMC) procedures, assuming a multivariate normal distribution (MVN) and using 50 imputed datasets in Stata 14.1. The results after multiple imputation were qualitatively consistent with the multivariable adjusted complete case analysis. 


\section{Results}

\section{Baseline characteristics}

Platelet counts were available for 642 melanoma patients. Patients with thrombocytosis were slightly younger and females were over-represented in this group. They also presented in more advanced stages, and subsequently were less likely to receive surgery and more likely to be treated with chemotherapy. There was less involvement of the head and neck areas in patients with thrombocytosis, approaching statistical significance. No differences in cardiovascular comorbidities were observed between the two groups (Table 1).

\section{Thrombocytosis and distant metastasis}

Given the established role of platelets in mediating metastasis in preclinical studies, the association between platelet count around time of diagnosis and distant metastasis (Stage IV) was investigated. Patients with thrombocytosis presented with more advanced stages (Table 1). Specifically, they were more likely to present in stage IV in univariate analysis and after adjusting for age and sex (Prevalence Ratio 3.5 [95\% Cl 2.35-5.22], Table 2). Analyzing platelets as a continuous variable showed that for every 100-unit increase in platelet count, patients were $33 \%$ (95\% Cl $21-47 \%)$ more likely to present with distant metastasis (Table 2). This association between thrombocytosis and distant metastasis persisted after sensitivity analysis using multiple imputation to account for patients with missing platelet counts and after including non-white patients (Supp. Tables 1, 2).

\section{Platelets predict overall survival}

Patients with thrombocytosis experienced shorter overall 5-year survival $(p=0.02$, Figure 1). Importantly, this decreased 5-year survival was most pronounced during the first year after diagnosis (Figure 1). Given these non-proportional hazards, we estimated the prognostic 
significance of platelets separately in the first year, and between 1 and 5 years for those alive at 1 year.

Higher platelet counts predicted increased mortality at 1 year in all stages of melanoma combined. This was also observed after adjusting for age, sex, stage and treatment modality (Table 3). Patients were then stratified into those with versus without distant metastatic disease (stages 0III versus IV). Thrombocytosis predicted shorter survival in stage IV within the first year, but not in patients with localized/regional spread (stages 0 -III, table 3). The same trend was observed when platelets were analyzed as a continuous variable, showing an incremental increase in mortality with each 100-unit increase of platelet counts. Among survivors at one year, platelet counts did not predict further survival in either metastatic or local/regional disease (Table 3). Upon performing sensitivity analysis to account for patients with no available platelet counts and to include the non-Caucasian population, similar associations were observed (Supp. Tables 3,4). Finally, further analysis of stages 0, I, II and III separately showed no predictive value of thrombocytosis; concordant with the pooled stage 0-III analysis (Supp. Table 5).

\section{Platelets promote melanoma dissemination to the lungs in mice}

To illustrate that targeting platelets is a viable option to prevent melanoma metastasis, we used a mouse model of thrombocytopenia[11]. The heat shock protein gp96 (HSP90b1) was selectively

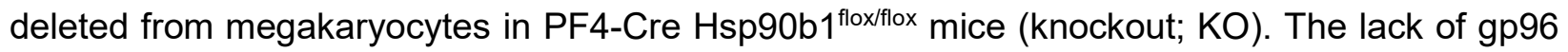
in megakaryocytes resulted in ineffective thrombopoiesis and low platelet counts (Figure 2A). Wild type (WT) and KO mice were injected with B16 melanoma cells via the lateral tail vein. Ten days later, $\mathrm{KO}$ mice had significantly fewer melanoma tumors in their lungs compared to their WT littermates (Figure 2B). 


\section{Discussion}

In this study, we observed that thrombocytosis is associated with melanoma distant metastasis and shorter overall survival. Within stage IV, higher platelet counts correlated with shorter survival in the first year in univariate analysis and after adjusting for age, sex, and treatment. These observations were consistent in sensitivity analyses to account for non-white patients and those with missing platelet counts. We used a mouse model of thrombocytopenia to show that targeting platelets protects against melanoma dissemination to the lungs. These data suggest that platelets could serve as a prognostic biomarker in patients with advanced melanoma, and that targeting this cellular entity can have a therapeutic role. It is worth mentioning that mortality from stage IV melanoma, especially prior to the institution of immunotherapy as a first line treatment, occurred largely in the first year after diagnosis, which likely explains the prognostic significance of platelets mainly in the first year, but not beyond.

The worse outcomes and more advanced stage associated with thrombocytosis is likely a product of two factors: Increased number of platelets propagates cancer dissemination and immune evasion, and intrinsically more aggressive cancers can incite systemic inflammation and induction of thrombopoiesis. This proposed bidirectional relationship is supported by evidence from preclinical studies as described in the introduction. Cancer is a chronic inflammatory state which often leads to secondary increase in thrombopoiesis due to inflammation or co-existent anemia of chronic disease, and platelets are in turn sabotaged in favor of the ongoing malignancy. In addition to their numbers, platelets are qualitatively altered in cancer patients, and were found to harbor tumor-derived RNA[19]. A recent study showed that increased platelet-to-lymphocyte ratio correlates with worse outcomes in melanoma, but did not independently evaluate the significance of platelet counts [20]. The study also was limited by a small sample size and not defining the cutoff value a priori. This cut-off was not validated in an independent patient cohort, which raises the concern about false positive observations. 
In this study, platelet counts were not available for all patients. It is conceivable that patients with more advanced stages are more likely to get blood tests including platelet counts as part of their work up. Indeed, around $70 \%$ of our patient population had Breslow thickness $>1 \mathrm{~mm}$, which is an over-representation. This is because blood counts are not part of the standard work up for thin melanomas. This limits the generalizability of our conclusions due to spectrum selection bias. To address that, we performed multiple imputation sensitivity analysis accounting for patients with no available platelet counts, and similar conclusions were obtained (Supp. Tables 1 and 3). Given that cardiovascular disease is the leading cause of death in the US population and the role of platelets in cardiovascular disease, we compared cardiovascular comorbidities across the two patient populations; there was no increase in cardiovascular comorbidities in patients with thrombocytosis (Table 1).

In a recent study, temporal measurement of platelet counts between four years prior to diagnosis and the time of treatment was used to predict outcomes in lung, prostate and colon cancers[21]. Adding platelet count features such as frequency of measurements among several other features increased the predictive value by $20-30 \%$ compared to post-diagnosis thrombocytosis alone. This study suggests a useful method of predicting outcomes, and provides deeper insight to the significance of platelets during the period of preclinical malignant transformation. Its applicability to everyday clinical practice has yet to be proven. The study was conducted on Veterans Affairs patients who mostly have their care in one centralized healthcare system. In contrast, non-veteran patients often seek medical care in different hospitals and outpatient settings, and are often referred to larger care centers for work up and management of their cancers. Hence, obtaining retrospective data on platelet counts can prove challenging. Nevertheless, the study is conceptually novel and with the aid of softwares tailored to capture platelet count features, such model could prove useful. 
In mouse models, we previously uncovered that platelets suppress anti-melanoma T cell immunity partly by activating TGF $\beta[11]$. Additionally, in the current study, we show that genetically targeting platelets in mice protects against melanoma dissemination to the lungs. In a separate clinical study, we observed that intake of aspirin correlated with longer overall survival in melanoma, particularly in stages II-IV[14]. Hence, targeting platelets is a promising adjuvant therapeutic approach in melanoma and cancer in general. Indeed, clinical trials are currently underway, combining platelet inhibitors such as aspirin and clopidogrel with immunotherapy in metastatic melanoma and head and neck carcinoma (NCT03396952 and NCT03245489).

\section{Acknowledgements}

We thank the Simon Cancer Center registry and the Clinical Data Warehouse at Indiana University Health for providing the patient data used in this study.

\section{Author Contributions}

SR conceived the idea. SR and MK designed the study. SR and TL performed data acquisition. MK performed data analysis. SR and ZL executed the mouse experiments. SR drafted the manuscript. MK, TL, and ZL reviewed the manuscript and provided critical feedback.

\section{Figures}

Figure 1. Overall survival in stages 0 -IV melanoma. 5-year survival from the time of diagnosis in melanoma patients of all stages $(0-\mathrm{IV}) . \mathrm{N}=642$, Log rank $\mathrm{p}=0.02$.

Figure 2. Platelet-specific knockout of gp96 results in thrombocytopenia and protection from melanoma lung colonization. A. Knockout mice were bled from the lateral tail vein to measure platelet counts. B. Mice were injected with $1 \times 10^{5} \mathrm{~B} 16$ melanoma cells in the lateral tail vein and mice were sacrificed 10 days later. Error bars represent standard error of the mean. Two-tailed independent Student t-test was used to compare the two groups. 


\section{References}

1. Shau H, Roth MD, Golub SH. Regulation of natural killer function by nonlymphoid cells. Natural immunity. 1993 Jul-Oct;12(4-5):235-49. PubMed PMID: 8257829; eng.

2. Nierodzik ML, Plotkin A, Kajumo F, et al. Thrombin stimulates tumor-platelet adhesion in vitro and metastasis in vivo. J Clin Invest. 1991 Jan;87(1):229-36. doi: 10.1172/jci114976. PubMed PMID: 1845869; PubMed Central PMCID: PMCPMC295033. eng.

3. Karpatkin S, Ambrogio C, Pearlstein E. The role of tumor-induced platelet aggregation, platelet adhesion and adhesive proteins in tumor metastasis. Prog Clin Biol Res. 1988;283:585-606. PubMed PMID: 3211961; eng.

4. Kaser A, Brandacher G, Steurer W, et al. Interleukin-6 stimulates thrombopoiesis through thrombopoietin: role in inflammatory thrombocytosis. Blood. 2001 Nov 1;98(9):2720-5. PubMed PMID: 11675343; eng.

5. Levin J, Conley CL. THROMBOCYTOSIS ASSOCIATED WITH MALIGNANT DISEASE. Archives of internal medicine. 1964 Oct;114:497-500. PubMed PMID: 14184638; eng.

6. Yu LX, Yan L, Yang W, et al. Platelets promote tumour metastasis via interaction between TLR4 and tumour cell-released high-mobility group box1 protein. Nature communications. 2014 Oct 28;5:5256. doi: 10.1038/ncomms6256. PubMed PMID: 25348021; eng.

7. Nieswandt B, Hafner M, Echtenacher B, et al. Lysis of tumor cells by natural killer cells in mice is impeded by platelets. Cancer Res. 1999 Mar 15;59(6):1295-300. PubMed PMID: 10096562; eng.

8. Palumbo JS, Talmage KE, Massari JV, et al. Platelets and fibrin(ogen) increase metastatic potential by impeding natural killer cell-mediated elimination of tumor cells. Blood. 2005 Jan 01;105(1):178-85. doi: 10.1182/blood-2004-06-2272. PubMed PMID: 15367435; eng.

9. Coupland LA, Chong BH, Parish CR. Platelets and P-selectin control tumor cell metastasis in an organ-specific manner and independently of NK cells. Cancer Res. 2012 Sep 15;72(18):4662-71. doi: 10.1158/0008-5472.can-11-4010. PubMed PMID: 22836751; eng.

10. Ward Y, Lake R, Faraji F, et al. Platelets Promote Metastasis via Binding Tumor CD97 Leading to Bidirectional Signaling that Coordinates Transendothelial Migration. Cell reports. $2018 \mathrm{Apr}$ 17;23(3):808-822. doi: 10.1016/j.celrep.2018.03.092. PubMed PMID: 29669286; eng.

11. Rachidi $S$, Metelli A, Riesenberg $B$, et al. Platelets subvert T cell immunity against cancer via GARP-TGFbeta axis. Sci Immunol. 2017 May 5;2(11). doi: 10.1126/sciimmunol.aai7911. PubMed PMID: 28763790; PubMed Central PMCID: PMCPMC5539882. eng.

12. Labelle M, Begum S, Hynes RO. Direct signaling between platelets and cancer cells induces an epithelial-mesenchymal-like transition and promotes metastasis. Cancer Cell. 2011 Nov 15;20(5):576-90. doi: 10.1016/j.ccr.2011.09.009. PubMed PMID: 22094253; PubMed Central PMCID: PMC3487108. eng.

13. Labelle M, Begum S, Hynes RO. Platelets guide the formation of early metastatic niches. Proc Natl Acad Sci U S A. 2014 Jul 29;111(30):E3053-61. doi: 10.1073/pnas.1411082111. PubMed PMID: 25024172; PubMed Central PMCID: PMCPMC4121772. eng.

14. Rachidi $\mathrm{S}$, Wallace $\mathrm{K}$, $\mathrm{Li} \mathrm{H}$, et al. Postdiagnosis aspirin use and overall survival in patients with melanoma. Journal of the American Academy of Dermatology. 2018 May;78(5):949-956.e1. doi: 10.1016/j.jaad.2017.12.076. PubMed PMID: 29317280; eng.

15. Moreau J, Pelletier F, Biichle S, et al. Increased levels of circulating platelet-derived microparticles are associated with metastatic cutaneous melanoma. Experimental dermatology. 2017 Oct;26(10):961-963. doi: 10.1111/exd.13339. PubMed PMID: 28266752; eng. 
16. Ikeda $\mathrm{M}$, Furukawa $\mathrm{H}$, Imamura $\mathrm{H}$, et al. Poor prognosis associated with thrombocytosis in patients with gastric cancer. Ann Surg Oncol. 2002 Apr;9(3):287-91. PubMed PMID: 11923136; eng.

17. Gucer F, Moser F, Tamussino K, et al. Thrombocytosis as a prognostic factor in endometrial carcinoma. Gynecol Oncol. 1998 Aug;70(2):210-4. doi: 10.1006/gyno.1998.5078. PubMed PMID: 9740692; eng.

18. Rachidi S, Wallace K, Day TA, et al. Lower circulating platelet count and antiplatelet therapy independently predict better outcomes in patients with head and neck squamous cell carcinoma. J Hematol Oncol. 2014 Sep 27;7(1):65. doi: 10.1186/s13045-014-0065-5. PubMed PMID: 25260646; PubMed Central PMCID: PMC4189675. Eng.

19. Nilsson RJ, Balaj L, Hulleman E, et al. Blood platelets contain tumor-derived RNA biomarkers. Blood. 2011 Sep 29;118(13):3680-3. doi: 10.1182/blood-2011-03-344408. PubMed PMID: 21832279; eng.

20. Qi Y, Zhang Y, Fu X, et al. Platelet-to-lymphocyte ratio in peripheral blood: A novel independent prognostic factor in patients with melanoma. International immunopharmacology. 2018 Mar;56:143-147. doi: 10.1016/j.intimp.2018.01.019. PubMed PMID: 29414644; eng.

21. Sylman JL, Boyce HB, Mitrugno A, et al. A Temporal Examination of Platelet Counts as a Predictor of Prognosis in Lung, Prostate, and Colon Cancer Patients. Scientific reports. $2018 \mathrm{Apr}$ 26;8(1):6564. doi: 10.1038/s41598-018-25019-1. PubMed PMID: 29700384; PubMed Central PMCID: PMCPMC5920102. eng. 
Table 1. Demographic and clinical characteristics of study population with platelet counts $(\mathrm{N}=642)$

\begin{tabular}{|c|c|c|c|c|}
\hline Variable & Overall & $\begin{array}{l}\text { Platelet count } \\
<350\end{array}$ & $\begin{array}{l}\text { Platelet count } \\
\geq 350\end{array}$ & $P$ value \\
\hline Mean age (SD), years & $57.7(16.1)$ & $58.1(16.3)$ & $53.0(13.4)$ & 0.03 \\
\hline Female, N (\%) & $270(42.1)$ & $237(40.2)$ & $33(63.5)$ & 0.001 \\
\hline $\begin{array}{l}\text { Stage, N (\%) } \\
\text { In situ } \\
\text { Stage I } \\
\text { Stage II } \\
\text { Stage III } \\
\text { Stage IV }\end{array}$ & $\begin{array}{l}154(24.0) \\
158(24.6) \\
83(12.9) \\
159(24.8) \\
88(13.7)\end{array}$ & $\begin{array}{l}145(24.6) \\
148(25.1) \\
79(13.4) \\
148(25.1) \\
70(11.9)\end{array}$ & $\begin{array}{l}9(17.3) \\
10(19.2) \\
4(7.7) \\
11(21.2) \\
18(34.6)\end{array}$ & $<0.001$ \\
\hline $\begin{array}{l}\text { Anatomical site, N (\%) } \\
\text { Head/neck } \\
\text { Trunk } \\
\text { Extremities } \\
\text { Other }\end{array}$ & $\begin{array}{l}146(22.7) \\
175(27.3) \\
247(38.5) \\
74(11.5)\end{array}$ & $\begin{array}{l}139(23.6) \\
163(27.6) \\
225(38.1) \\
63(10.7)\end{array}$ & $\begin{array}{l}7(13.5) \\
12(23.1) \\
22(42.3) \\
11(21.2)\end{array}$ & 0.06 \\
\hline Diabetes mellitus, N (\%) & $108(16.8)$ & $102(17.3)$ & $6(11.5)$ & 0.29 \\
\hline Hypertension, N (\%) & $225(35.0)$ & $211(35.8)$ & $14(26.9)$ & 0.20 \\
\hline Dyslipidemia, N (\%) & $189(29.4)$ & $177(30.0)$ & $12(23.1)$ & 0.29 \\
\hline Cardiovascular disease, N (\%) & $76(11.8)$ & $73(12.4)$ & $3(5.8)$ & 0.16 \\
\hline Surgery, N (\%) & $563(87.7)$ & $528(89.5)$ & $35(67.3)$ & $<0.001$ \\
\hline Chemotherapy, N (\%) & $45(7.1)$ & $37(6.3)$ & $8(15.4)$ & 0.02 \\
\hline Radiation, N (\%) & $49(7.6)$ & $45(7.6)$ & $4(7.7)$ & 0.99 \\
\hline Immunotherapy, N (\%) & $73(11.4)$ & $70(11.9)$ & $3(5.8)$ & 0.18 \\
\hline $\begin{array}{l}\text { Breslow thickness } \geq 1 \mathrm{~mm}, \mathrm{~N} \\
(\%)\end{array}$ & $236(69.2)$ & $219(69.1)$ & $17(70.8)$ & 0.86 \\
\hline
\end{tabular}

Fisher's exact tests were used in place of Chi-square test when cell sizes were $<10$. 
Table 2. Prevalence ratios and $95 \%$ confidence intervals for association between platelet counts and stage IV melanoma (versus stage $0-\mathrm{III}, \mathrm{N}=642$ )

\begin{tabular}{|l|l|l|l|l|}
\hline Exposure & \multicolumn{2}{|l|}{ Platelets $\mathbf{2 3 5 0}$} & Platelets $^{\mathbf{b}}$ & \\
\hline & PR (95\% Cl) & P & PR (95\% Cl) & P \\
\hline Unadjusted & $2.92(1.89,4.50)$ & $<0.001$ & $1.37(1.26,1.50)$ & $<0.001$ \\
\hline Adjusted $^{a}$ & $3.50(2.35,5.22)$ & $<0.001$ & $1.33(1.21,1.47)$ & $<0.001$ \\
\hline
\end{tabular}

${ }^{a}$ Adjusted for age and sex

bPlatelets as a continuous variable was modeled for a 100-unit increase 
Table 3. Hazard ratios and 95\% confidence intervals for platelet counts and survival in melanoma.

\begin{tabular}{|c|c|c|c|c|}
\hline \multirow[t]{2}{*}{ Exposure } & \multicolumn{2}{|c|}{ Platelets $\geq 350$} & \multicolumn{2}{|c|}{ Platelets $^{c}$} \\
\hline & HR (95\% Cl) & $\mathbf{P}$ & HR (95\% Cl) & $\mathbf{P}$ \\
\hline \multicolumn{5}{|c|}{ 1-year survival } \\
\hline \multicolumn{5}{|c|}{ All stages combined $(\mathrm{N}=642)$} \\
\hline Unadjusted & $3.24(1.84,5.69)$ & $<0.001$ & $1.52(1.33,1.72)$ & $<0.001$ \\
\hline Adjusted $^{a}$ & $1.92(1.08,3.43)$ & 0.03 & $1.29(1.13,1.48)$ & $<0.001$ \\
\hline \multicolumn{5}{|c|}{ Stages 0 -III $(\mathrm{N}=554)$} \\
\hline Unadjusted & $0.85(0.11,6.39)$ & 0.88 & $1.31(0.72,2.38)$ & 0.38 \\
\hline Adjusted $^{a}$ & $1.18(0.15,9.03)$ & 0.87 & $1.63(0.89,3.00)$ & 0.12 \\
\hline \multicolumn{5}{|c|}{ Stage IV $(\mathbf{N}=\mathbf{8 8})$} \\
\hline Unadjusted & $1.74(0.95,3.17)$ & 0.07 & $1.14(0.99,1.30)$ & 0.05 \\
\hline Adjusted $^{\mathrm{b}}$ & $1.80(0.97,3.34)$ & 0.06 & $1.20(1.04,1.40)$ & 0.01 \\
\hline \multicolumn{5}{|c|}{ 5-year survival in patients alive at 1 year } \\
\hline \multicolumn{5}{|c|}{ All stages combined $(\mathrm{N}=553)$} \\
\hline Unadjusted & $0.63(0.23,1.73)$ & 0.37 & $0.84(0.62,1.15)$ & 0.28 \\
\hline Adjusted $^{\mathrm{a}}$ & $0.45(0.16,1.25)$ & 0.13 & $0.85(0.62,1.16)$ & 0.31 \\
\hline \multicolumn{5}{|c|}{ Stages 0 -III $(N=523)$} \\
\hline Unadjusted & $0.62(0.20,1.98)$ & 0.42 & $0.81(0.57,1.15)$ & 0.24 \\
\hline Adjusted $^{a}$ & $0.99(0.30,3.24)$ & 0.98 & $1.05(0.71,1.56)$ & 0.81 \\
\hline \multicolumn{5}{|c|}{ Stage IV ( $\mathbf{N}=\mathbf{3 0})$} \\
\hline Unadjusted & $0.34(0.04,2.56)$ & 0.29 & $0.82(0.46,1.45)$ & 0.49 \\
\hline Adjusted $^{b}$ & $0.30(0.04,2.34)$ & 0.25 & $0.74(0.41,1.34)$ & 0.33 \\
\hline
\end{tabular}

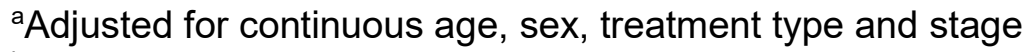

${ }^{\mathrm{b} A d j u s t e d}$ for continuous age, sex and treatment type

'Platelets as a continuous variable was modeled for a 100-unit increase 


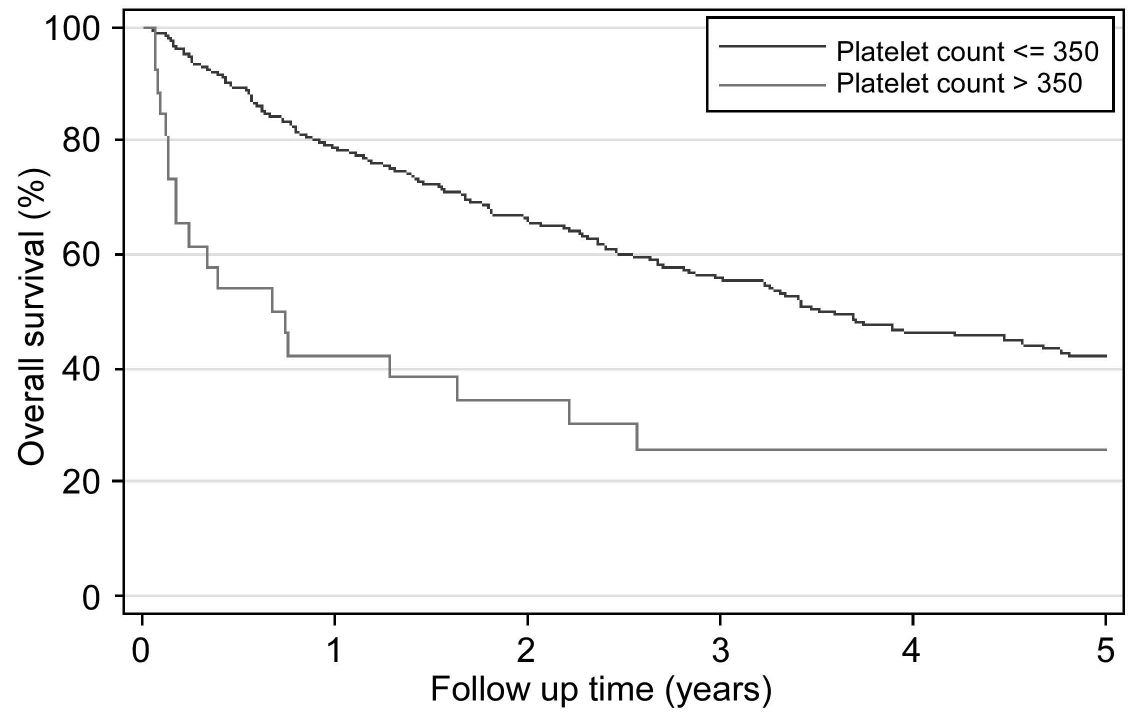




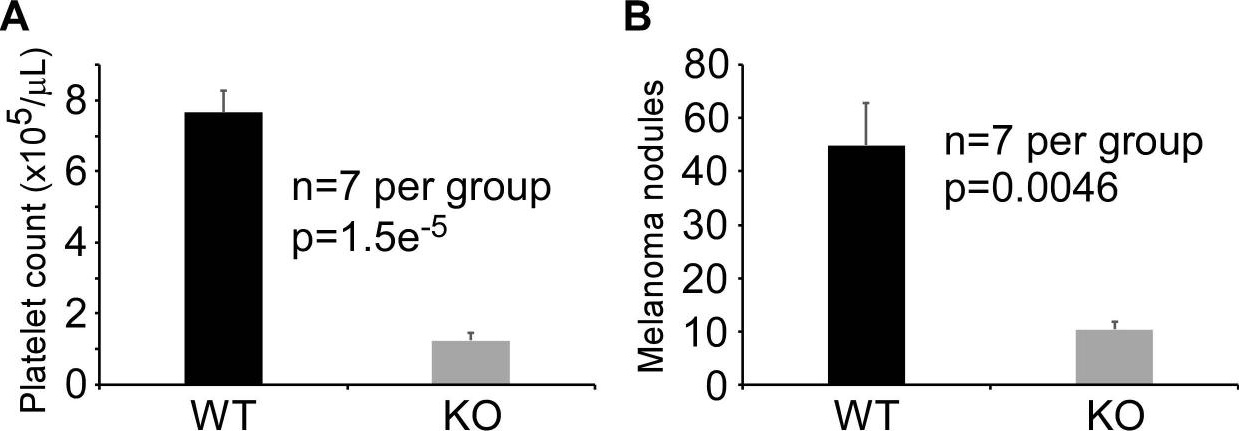




\section{Supplementary material. Rachidi et al. 2018}

Table S1. Sensitivity analysis of prevalence ratios and 95\% confidence intervals for association between platelet count and stage IV melanoma (versus stages 0 -III) using multiple imputation to replace missing platelet counts

\begin{tabular}{|l|l|l|l|l|}
\hline Exposure & \multicolumn{2}{|l|}{ Platelets $\geq 350$} & Platelets \\
\hline & PR $(95 \% \mathrm{Cl})$ & $\mathrm{P}$ & $\mathrm{PR}(95 \% \mathrm{Cl})$ & $\mathrm{P}$ \\
\hline Unadjusted & $1.95(1.17,3.24)$ & 0.01 & $1.37(1.21,1.55)$ & $<0.001$ \\
\hline Adjusted $^{\mathrm{a}}$ & $2.41(1.43,4.09)$ & 0.001 & $1.39(1.25,1.55)$ & $<0.001$ \\
\hline
\end{tabular}

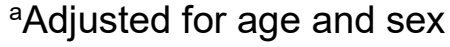

bPlatelets as a continuous variable was modeled for a 100-unit increase

Table S2. Sensitivity analysis of prevalence ratios and 95\% confidence intervals for association between platelet count and stage IV melanoma (versus stages 0 -III) including non-Caucasian patients $(\mathrm{N}=655)$

\begin{tabular}{|l|l|l|l|l|}
\hline Exposure & \multicolumn{2}{|l|}{ Platelets $\geq 350$} & \multicolumn{2}{l|}{ Platelets $^{\text {b }}$} \\
\hline & PR $(95 \% \mathrm{Cl})$ & $\mathrm{P}$ & $\mathrm{PR}(95 \% \mathrm{Cl})$ & $\mathrm{P}$ \\
\hline Unadjusted & $2.80(1.82,4.32)$ & $<0.001$ & $1.37(1.25,1.49)$ & $<0.001$ \\
\hline Adjusted $^{\text {a }}$ & $3.29(2.19,4.94)$ & $<0.001$ & $1.33(1.21,1.46)$ & $<0.001$ \\
\hline
\end{tabular}

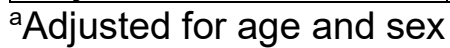

bPlatelets as a continuous variable was modeled for a 100-unit increase

Table S3. Sensitivity analysis of hazard ratios and 95\% confidence intervals for platelet count and survival using multiple imputation to replace missing platelet counts

\begin{tabular}{|c|c|c|c|c|}
\hline \multirow[t]{2}{*}{ Exposure } & \multicolumn{2}{|c|}{ Platelets $\geq 350$} & \multicolumn{2}{|c|}{ Platelets $^{\mathrm{c}}$} \\
\hline & $\mathrm{HR}(95 \% \mathrm{Cl})$ & $\mathrm{P}$ & $\mathrm{HR}(95 \% \mathrm{Cl})$ & $\mathrm{P}$ \\
\hline \multicolumn{5}{|c|}{ 1-year survival } \\
\hline \multicolumn{5}{|c|}{ All stages combined } \\
\hline Unadjusted & $1.85(0.99,3.45)$ & 0.05 & $1.44(1.21,1.72)$ & $<0.001$ \\
\hline Adjusted $^{a}$ & $1.68(0.94,3.00)$ & 0.08 & $1.31(1.13,1.50)$ & $<0.001$ \\
\hline \multicolumn{5}{|l|}{ Stages 0-III } \\
\hline Unadjusted & $0.74(0.12,4.39)$ & 0.74 & $1.04(0.62,1.74)$ & 0.88 \\
\hline Adjusted $^{a}$ & $1.04(0.17,6.34)$ & 0.97 & $1.31(0.80,2.17)$ & 0.28 \\
\hline \multicolumn{5}{|l|}{ Stage IV } \\
\hline Unadjusted & $1.47(0.79,2.73)$ & 0.22 & $1.14(0.99,1.31)$ & 0.06 \\
\hline Adjusted $^{b}$ & $1.55(0.82,2.91)$ & 0.18 & $1.21(1.04,1.42)$ & 0.02 \\
\hline \multicolumn{5}{|c|}{ 5-year survival in patients alive at 1 year } \\
\hline \multicolumn{5}{|c|}{ All stages combined } \\
\hline Unadjusted & $0.79(0.39,1.61)$ & 0.51 & $0.91(0.70,1.18)$ & 0.49 \\
\hline Adjusted $^{\mathrm{a}}$ & $0.80(0.38,1.69)$ & 0.56 & $0.98(0.74,1.28)$ & 0.87 \\
\hline \multicolumn{5}{|l|}{ Stages 0 -III } \\
\hline Unadjusted & $0.77(0.34,1.71)$ & 0.52 & $0.89(0.67,1.18)$ & 0.42 \\
\hline Adjusted $^{a}$ & $1.12(0.49,2.58)$ & 0.79 & $1.14(0.86,1.51)$ & 0.35 \\
\hline
\end{tabular}




\begin{tabular}{|l|l|l|l|l|}
\hline Unadjusted & $0.55(0.11,2.90)$ & 0.48 & $0.91(0.54,1.54)$ & 0.72 \\
\hline Adjusted $^{\mathrm{b}}$ & $0.53(0.10,2.82)$ & 0.46 & $0.86(0.50,1.48)$ & 0.59 \\
\hline
\end{tabular}

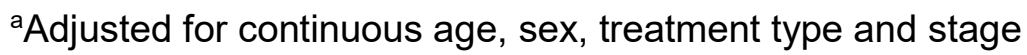

${ }^{\mathrm{b} A d j u s t e d}$ for continuous age, sex and treatment type

'Platelets as a continuous variable was modeled for a 100-unit increase

Table S4. Sensitivity analysis of platelet count and survival including non-Caucasian patients $(\mathrm{N}=655)$

\begin{tabular}{|c|c|c|c|c|}
\hline Exposure & Platelets $\geq 350$ & & Platelets $^{\mathrm{b}}$ & \\
\hline & $\mathrm{HR}(95 \% \mathrm{Cl})$ & $P$ & $\mathrm{HR}(95 \% \mathrm{Cl})$ & $P$ \\
\hline 1-year survi & & & & \\
\hline Unadjusted & $3.34(1.93,5.78)$ & $<0.001$ & $1.52(1.34,1.72)$ & $<0.001$ \\
\hline Adjusted $^{a}$ & $2.03(1.15,3.56)$ & 0.001 & $1.28(1.12,1.46)$ & $<0.001$ \\
\hline 5-year surv & U1 1-yeal & vival & & \\
\hline Unadjusted & $0.64(0.23,1.76)$ & 0.39 & $0.84(0.61,1.14)$ & 0.27 \\
\hline Adjusted $^{\mathrm{a}}$ & $0.45(0.16,1.27)$ & 0.13 & $0.84(0.61,1.15)$ & 0.27 \\
\hline
\end{tabular}

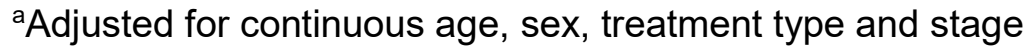

bPlatelets as a continuous variable was modeled for a 100-unit increase

Table S5. Hazard ratios and 95\% confidence intervals for platelet count and survival stratified by stage.

\begin{tabular}{|c|c|c|}
\hline \multirow[t]{2}{*}{ Exposure } & \multicolumn{2}{|l|}{ Platelets $\geq 350$} \\
\hline & $\mathrm{HR}(95 \% \mathrm{Cl})$ & $\mathrm{P}$ \\
\hline \multicolumn{3}{|c|}{ Stage $0(\mathrm{~N}=154), 5$-year survival ${ }^{\mathrm{a}}$} \\
\hline Unadjusted & $1.20(0.16,9.22)$ & 0.86 \\
\hline Adjusted $^{b}$ & $2.40(0.27,21.13)$ & 0.43 \\
\hline \multicolumn{3}{|c|}{ Stage I $(\mathrm{N}=158), 5$-year survival ${ }^{a}$} \\
\hline Unadjusted & $0.90(0.12,6.90)$ & 0.92 \\
\hline Adjusted $^{b}$ & $1.59(0.19,13.18)$ & 0.67 \\
\hline \multicolumn{3}{|c|}{ Stage II ( $\mathrm{N}=83), 5$-year survival ${ }^{\mathrm{c}}$} \\
\hline Unadjusted & - & - \\
\hline Adjusted & - & - \\
\hline \multicolumn{3}{|l|}{ Stage III } \\
\hline \multicolumn{3}{|c|}{ 1-year survival $(\mathrm{N}=159)$} \\
\hline Unadjusted & $1.77(0.22,14.19)$ & 0.59 \\
\hline Adjusted $^{\mathrm{d}}$ & $1.95(0.23,16.68)$ & 0.54 \\
\hline \multicolumn{3}{|c|}{5 -year survival in patients alive at 1 year $(\mathrm{N}=148)$} \\
\hline Unadjusted & $0.43(0.06,3.14)$ & 0.40 \\
\hline Adjusted $^{d}$ & $0.60(0.08,4.69)$ & 0.63 \\
\hline
\end{tabular}




\begin{tabular}{|l|l|l|}
\hline 1-year survival $(\mathrm{N}=88)$ & 0.07 \\
\hline Unadjusted $^{\mid} 1.74(0.95,3.17)$ & 0.06 \\
\hline Adjusted $^{\mathrm{d}}$ & $1.80(0.97,3.34)$ & \\
\hline 5-year survival in patients alive at 1 year $(\mathrm{N}=30)$ & 0.29 \\
\hline Unadjusted $^{\mathrm{d}}$ & $0.34(0.04,2.56)$ & 0.25 \\
\hline Adjusted $^{\mathrm{d}}$ & $0.30(0.04,2.34)$ & \\
\hline
\end{tabular}

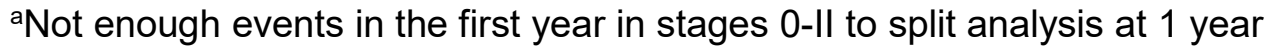
${ }^{b}$ Adjusted for continuous age and sex. Patients uniformly treated surgically ${ }^{\circ}$ Only 4 patients in thrombocytosis group, none dead. Hence, survival analysis not possible ${ }^{\mathrm{d} A d j u s t e d ~ f o r ~ c o n t i n u o u s ~ a g e, ~ s e x ~ a n d ~ t r e a t m e n t . ~}$ 